
Recoived tu $\cap 07$ GA-A20107

OCT 221990

\title{
FAST ALPHA DIAGNOSTICS USING CARBON PELLET INJECTION
}

\author{
by \\ R.K. FISHER, J.M. MCCHESNEY, A.M. HOWALD, \\ P.B. PARKS, D.M. THOMAS, S.C. MCCOOL, \\ and W.L. ROMAN
}

SEPTEMBER 1990

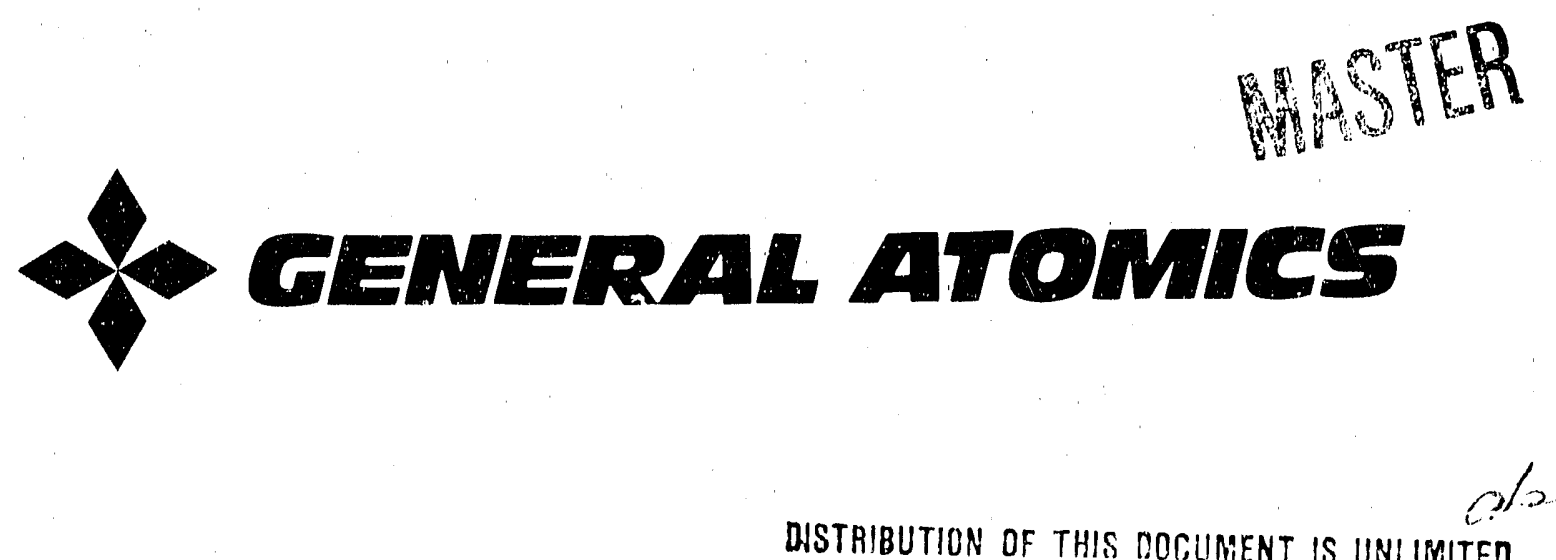




\section{DISCLAIMER}

This report was prepared as an account of work sponsored by an agency of the United States Governmenit. Neither the United States Covernment nor any agency thereof, nor any of their employees, rakkes any warranty, express or implied, or assumes any legal liability or responsibility for the accuracy, completeness, or usefulness of any information, apparatus, prociuct, or process disclosed, or represents that its use would not infringe privately ovjned rights. Reference herein to any specific commercial product, process, or service by trade nanie, trademark, nlanufacturer, or otherwise, does not necessarily constitute or imply its endorsement, recommendation, or favoring by the United States Government or any agency thereof. The views and opinions of authors expressed herein do not neseswarily state or reflect those of the United States Government or any agency thereof. 


\title{
FAST ALPHA DIAGNOSTICS USING CARBON PELLET INJECTION
}

\author{
by \\ R.K. FISHER, J.M. MCCHESNEY, A.M. HOWALD, \\ P.B. PARKS, D.M. THOMAS, S.C. McCOOL, \\ and W.L. ROMAN*
}

This is a preprint of a paper to be presented at the Eighth Topical Conference on High-Temperature Plasma Diagnostics, May 7-10, 1990, in Hyannis, Massachusetts and to be printed in the Proceedings.

\author{
Work supported by \\ U.S. Department of Energy \\ Contract DE-AC03-89ER53277
}

*University of Texas at Austin, Austin, Texas.

GENERAL ATOMICS PROJECT 3468

SEPTEMBER 1990 


\title{
Fast alpha diagnostics using carbon pellet injection
}

R.K. Fisher, J.M. McChesney, A.M. Howald, P.B. Parks, D.M. Thomas, S.C. McCool,* and W.L. Rowan*

General Atomics, San Diego, California 92186-9084

*University of Texas at Austin, Austin, Texas

\begin{abstract}
Charge exchange interactions of alpha particles with the ablation cloud surrounding an injected carbon pellet can be used to measure the energy spectrum of the incident fast confined alpha particles in a fusion plasma. Measurement of helium neutrals from $\mathrm{He}^{2+}+\mathrm{C}^{4+} \rightarrow \mathrm{He}^{0}+\mathrm{C}^{6+}$ interactions appears to be the most attractive option. This paper describes progress on developing this diagnostic including measurements during pellet injection into the TEXT tokamak.
\end{abstract}




\section{INTRODUCTION}

Obtaining information on the physics of alpha particle heating will be one of the major goals of the upcoming deuterium-tritium experiments planned for TFTR, JET, and CIT. These experiments will offer the first opportunity to study the confinement and interactions of alpha particles in a fusion plasma.

It will not be easy to measure the properties of the fast confined alpha particles in a tokamak: Several reviews and workshops have addressed this topic. ${ }^{1-4}$ We have proposed the idea of using impurity pellet injection to provide a target for double charge exchange interartions of fast alpha particles with the ablation cloud surrounding an injected impurity pellet. ${ }^{5}$ This technique offers a direct measurement of the energy distribution of incident-high energy alphas. Since 1988, we have been developing an alpha diagnostic based on carbon pellet injection. This paper will describe progress on this diagnostic.

\section{DOUBLE CHARGE EXCHANGE TE :HNIQUE}

A small fraction of the alpha particles incident on the pellet ablation cloud will be converted to helium neutrals. Carbon is an attractive pellet material because it penetrates well due to its high heat of ablation and because it has favorable atomic physics properties. The double charge exchange reaction

$$
\mathrm{He}^{++}+\mathrm{C}^{4+} \rightarrow \mathrm{He}^{\circ}+\mathrm{C}^{\mathrm{E}+}
$$

is of particular interest because a large region of the pellet ablation cloud will be dominated by the $\mathrm{C}^{4+}$ ionization state as discussed in Section III. The density in the $\mathrm{C}^{4+}$ region of the cloud should be high enough to produce an equilibrium fraction $\mathrm{F}_{0}^{\infty}$ of helium neutrals. Hence, the measured neutral distribution $d n_{\mathrm{He}^{++}} / d \mathrm{E}$ is related to the incident alpha distribution $d n_{\mathrm{He}^{++}} / d \mathrm{E}$ by 


$$
\frac{d n_{\mathrm{He}^{\mathrm{o}}}}{d \mathrm{E}}=\frac{d n_{\mathrm{He}^{++}}}{d \mathrm{E}} \quad F_{0}^{\infty}(\mathrm{E}, Z=4)
$$

The production of neutrals is dominated by the double charge exchange cross section $\sigma_{20}(\mathrm{E}, Z=4)$ while the dominant loss mechanism is impact reionization

$$
\mathrm{He}^{0}+\mathrm{C}^{4+} \rightarrow \mathrm{He}^{+}+\mathrm{e}^{--}+\mathrm{C}^{4+},
$$

with cross section $\sigma_{01}(\mathrm{E}, Z=4)$. These two cross sections dominate the calculation ${ }^{\mathrm{B}}$ of

$$
\mathrm{F}_{0}^{\infty}(\mathrm{E}, Z=4) \simeq \frac{\sigma_{20}(\mathrm{E}, Z=4)}{\sigma_{01}(\mathrm{E}, Z=4)}
$$

From measurements of $\sigma_{01}(\mathrm{E})$ and calculations ${ }^{7}$ of $\sigma_{20}(\mathrm{E})$, we can determine $\mathrm{F}_{0}^{\infty}(\mathrm{E}, Z=4)$. Hence, measurements of the energy spectrum of helium neutrals reaching the plasma edge $d n_{\mathrm{He}^{\circ}} / d \mathrm{E}$ and use of Eq. (1) should allow determination of the incident fast alpha energy spectrum $d n_{\mathrm{He}^{++}} / d \mathrm{E}$.

\section{PELLET PENETRATION AND ABLATION}

In order to determine the pellet velocity needed to penetrate TFTR and CIT, we have developed an impurity pellet ablation model. Details of this model have been published elsewhere. ${ }^{8}$ The model includes shielding of the pellet surface because the ablation cloud absorbs a portion of the incident electron heat loading. Other sources of ablation, including alphas and plasma and neutral beam injection ions, are only small corrections.

The distance a pellet will penetrate depends on its size, velocity, and composition. If the pellet size is linited to contain less than $1 \%$ of the total number of plasma particles to limit the perturbations to the plasma, pellet penetration will be difficult. Figure 1 shows the velocity needed for a $1.0 \mathrm{~mm}$ diameter carbon pellet 
$\left(\mathrm{N}_{\text {pellet }} / \mathrm{N}_{\text {plasma }} \sim 10^{-2}\right)$ to penetrate CIT with $n_{e 0}=10^{15} \mathrm{~cm}^{-3}$ and $\mathrm{T}_{e 0}=20 \mathrm{keV}$. The horizontal axis is the penetration radius in units of the CIT horizontal minor radius of $65 \mathrm{~cm}$. The two curves show the needed velocity under the assumption of "peaked" and "broad" density profiles used in CIT planning. While a pellet velocity of $20 \mathrm{~km} / \mathrm{sec}$ is needed to penetrate to the center of CIT, it only takes $5 \mathrm{~km} / \mathrm{sec}$ to penetrate more than half way into CIT. Two-stage gas guns at ORNL have achieved almost $5 \mathrm{~km} / \mathrm{sec}$ and should be able to reach $7 \mathrm{~km} / \mathrm{sec}^{9}{ }^{9} \mathrm{~A} 100 \mathrm{kV}$ electric gun at LLNL has accelerated small flat projectiles to greater than $20 \mathrm{~km} / \mathrm{sec}^{10}$

The predicted velocities needed for CIT may be overestimated due to the simplicity of the model used to calculate the cloud shielding. The model assumes spherical expansion of the cloud while the tokamak magnetic field will, beyond some radius, force the cloud expansion to be channeled along the direction of the magnetic field. Since the plasma electron gyroradius is small compared to the pellet size, the electron heat flow ablating the pellet arrives along the magnetic field lines and will have to penetrate this elongated and thicker pellet ablation cloud. Measurements on TEXT indicate the ionized ablation cloud is channeled along the field and is traveling along with the pellet rather than being left behind tied to the field lines. This is thought due to the $\overline{\mathrm{E}} \times \overline{\mathrm{B}}$ drift of the cloud as a charge separation is built up due to the $\bar{v} \times \overline{\mathrm{B}}$ force, resulting in a cloud drift velocity just equal to the pellet velocity. If the cloud ablation continued to drift along with the pellet forever, the shielding would eventually prevent the electron heat load from reaching the pellet surface. Experimentally, we observe that the pellet fully ablates, so that at some distance from the pellet, the ablation material must start to lag behind the pellet. The shielding could be significantly better than our existing code predicts, however, particularly in tokamaks where strong magnetic channeling is expected, such as CIT.

Pellet penetration data taken on TEXT and TFTR is somewhat inconsistent. The observed penetration of carbon pellets into TEXT is usually less than our pellet ablation model predicts, sometimes by a very significant amount. Data on TFTR is, 


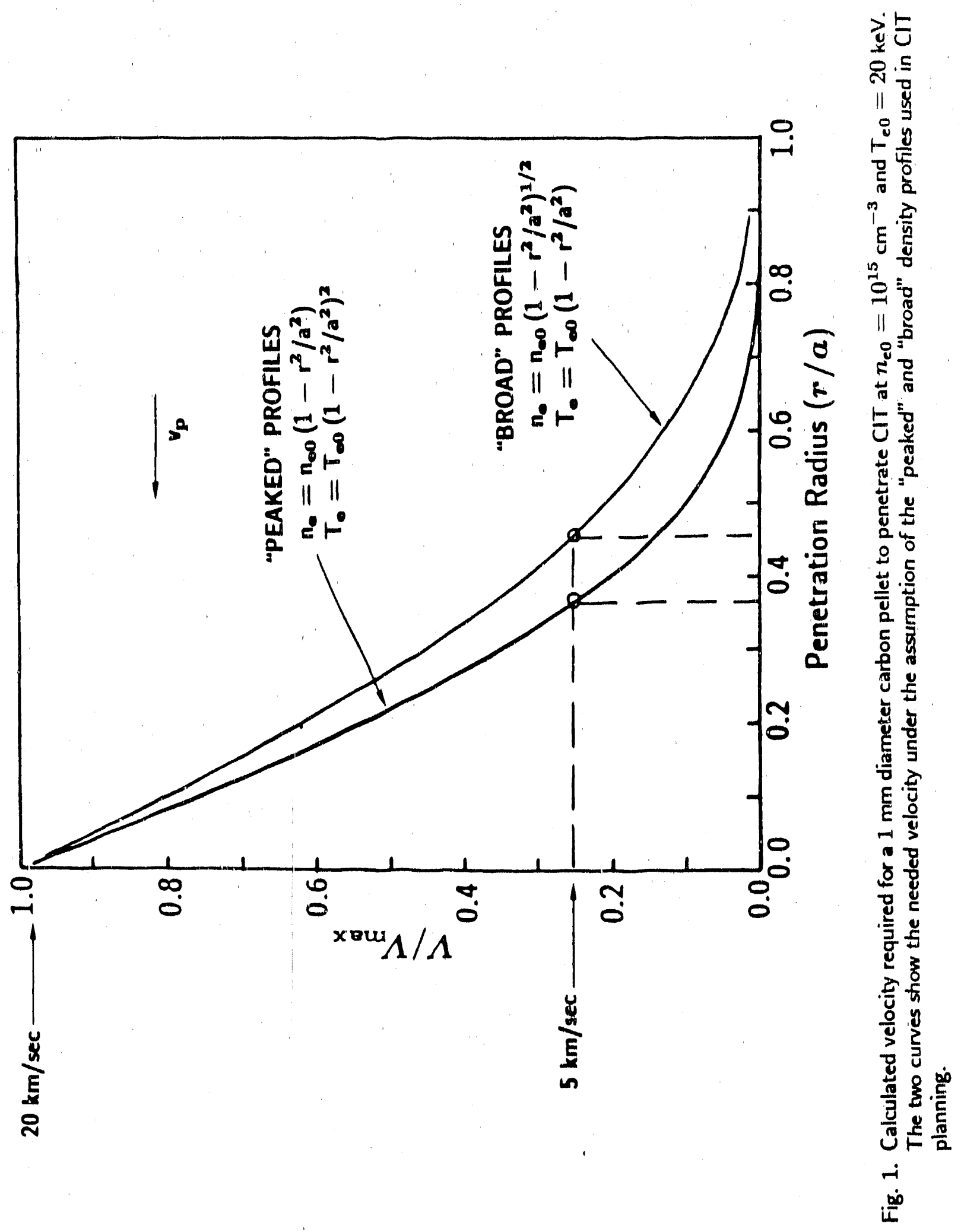


in general, in better agreement with the model. ${ }^{11}$ Since the TFTR plasma parameters and pellet size are closer to those planned for CIT, we are encouraged that the TFTR results agree reasonably well with the code.

\section{CHARGE STATE DISTRIBUTION}

In order to determine $d n_{\mathrm{He}^{+}+} / d \mathrm{E}$ from Eq. (1), we need to verify that a large spatial region of the cloud will be predominantly $\mathrm{C}^{4+}$ ions. This result is expected due to the large jump in the ionization potential from $\mathrm{C}^{3+}\left(\chi_{i} \sim 64 \mathrm{eV}\right)$ to $\mathrm{C}^{k+}\left(\chi_{i}=392 \mathrm{eV}\right)$. As the density falls off and the temperature rises at increasing distance from the pellet surface, we expect a progression to higher and higher ionization states beginning with neutral carbon coming off the pellet surface. Saha or coronal equilibrium would predict that a given charge state exists where the cloud electron temperature is of the order of a tenth of its ionization potential. Hence, there should be a large region of $\mathrm{C}^{4+}$ with very little $\mathrm{C}^{3+}$ or $\mathrm{C}^{5+}$ extending over a distance of several centimeters from the cloud. Work on modeling the spatial distribution of the charge states is in progress at Old Dominion University ${ }^{12}$ and at GA.

One of the major goals of our experiment on TEXT is to obtain data on the spatial distributions of different ionization states of carbon surrounding an injected carbon pellet. Figure 2 shows the spatial distribution of $5801 \AA$ line radiation from $\mathrm{C}^{3+}$ measured with a Xybion image intensified camera. This is a multiple exposure with the pellet traveling in the direction shown. The camera is gated on for $10 \mu \mathrm{sec}$ every $50 \mu \mathrm{sec}$ to obtain the cloud pictures at different plasma minor radii. The cloud is spread out along the magnetic field direction as expected. The two peaks in the $\mathrm{C}^{3+}$ intensity are separated by a region close to the pellet where the carbon is in lower ionization states.

The distance from the pellet to the peaks (assumed to be one-half the distance between peaks in Fig. 2) was found to be a strong function of the plasma electron density in TEXT. The data in Fig. 2 was taken at $n_{\mathrm{eo}}=2.6 \cdot 10^{19} \mathrm{~cm}^{-3}$. The $\mathrm{C}^{3+}$ 


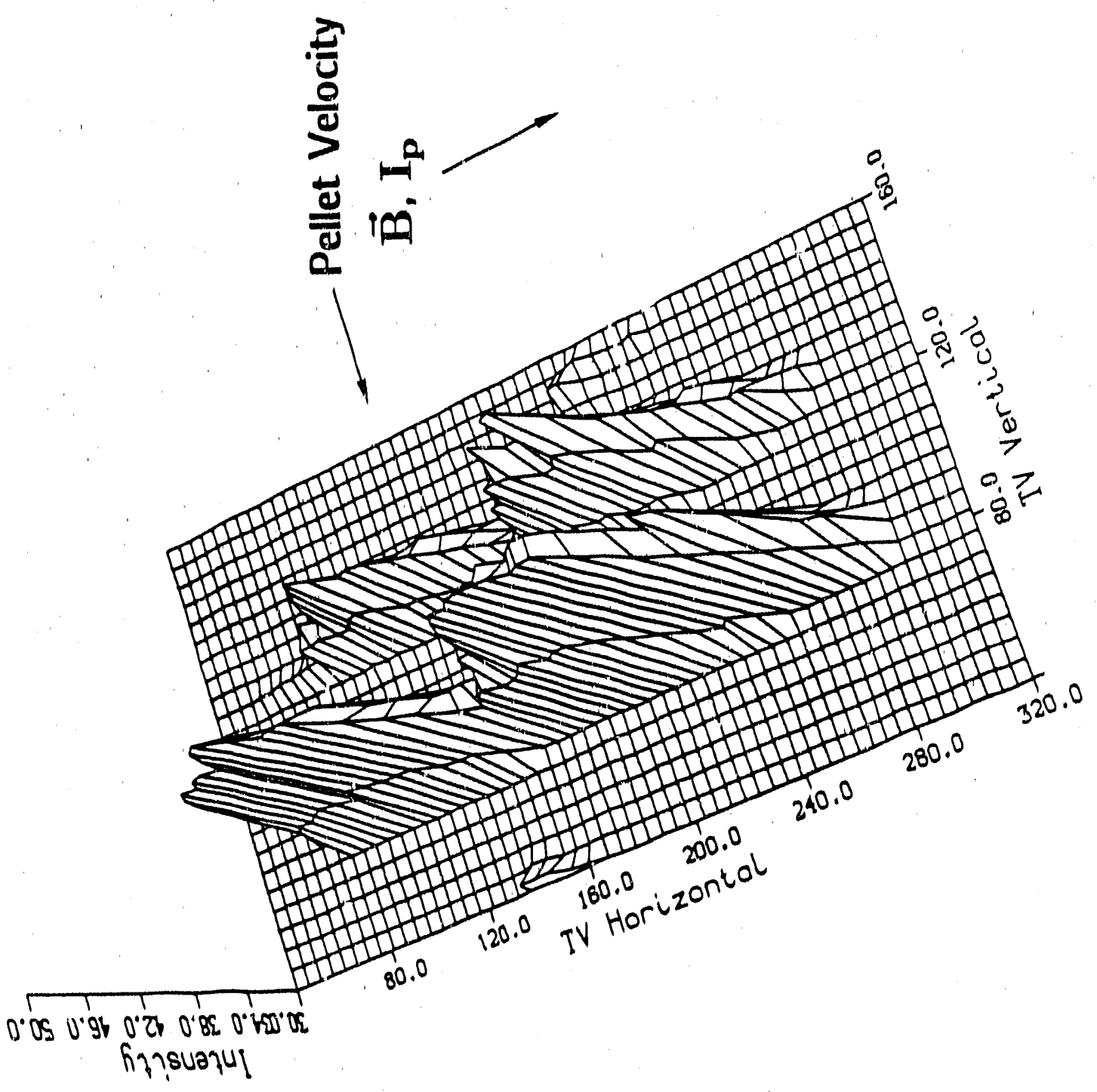

Fig. 2. Intensity contour plot from multiple exposure picture of $\mathrm{C}^{9+}$ light from cartson pellet injected into TEXT at $n_{e 0}=2.6 \cdot 10^{13} \mathrm{~cm}^{-3}$. 
light peaks about $2 \mathrm{~cm}$ frorn the pellet and the total cloud width is about $8 \mathrm{~cm}$. At a central density of $4.4 \cdot 10^{13} \mathrm{~cm}^{-3}$, the distance from the pellet to the $\mathrm{C}^{3+}$ peaks decreased to only $0.3 \mathrm{~cm}$ and the total cloud width is about $1.5 \mathrm{~cm}$. The reason for this strong density dependence is not yet clear. One possible explanation is based on the idea that as $n_{e 0}$ increases, the cloud channel width decreases, curtailing the expansion cooling effect. This, in turn, elevates the temperatu:e and thermal ionization rate of the cloud with the result that the distance from the pellet to a given charge state may become significantly smaller.

We have also observed $\mathrm{C}^{2+}$ light at $2297 \AA$. The $\mathrm{C}^{2+}$ light appears to peak closer to the pellet as expected, with only a small drop between maxima in the toroidal field direction.

$\mathrm{C}^{4+}$ is expected to exist outside the $\mathrm{C}^{3+}$ peaks and extend considerably further from the pellet due to the jump in ionization potential. Unfortunately, there were not any lines of $\mathrm{C}^{4+}$ bright enough to see with our $\mathrm{Xybion}$ image-intensified camera. The camera is only sensitive above $3800 \AA$ and higher ionization states have their strongest lines in the ultraviolet. We tried using a UV-to-visible wavelength converter in front of the camera. The scintillator tried was tetraphenyl butadiene (TPB). We were unable to see the $2271 \AA$ line of $C^{4+}$ but did see the $2297 \AA$ line of $\mathrm{C}^{2+}$ previously mentioned. We also tried many other visible lines of $\mathrm{C}^{5+}$ and lower ionization states but these signals all appeared to be dominated by bremsstrahlung continuum from the pellet cloud passing through the $10 \AA$ wide interference filters. The $\mathrm{C}^{3+}$ region of the cloud lies outside the $\mathrm{C}^{2+}$ region and is larger in spatial extent in the toroidal field direction. This is consistent with our expectation that there will be a large region of the cloud dominated by $\mathrm{C}^{4+}$ occurring at larger distances from the pellet than the observed $\mathrm{C}^{3+}$ light. It is premature at this stage to make more quantitative predictions of where a given ionization state should peak since the model describing the atomic rate processes of innization and recombination in an anisotropic expanded ablation cloud is incomplete. Work is continuing in developing such a model. Ionization by impact 
of plasma electrons and electrons arising from ionization of the cloud can both be shown to be important sources of ionization.

Given the value of being able to directly observe $\mathrm{C}^{4+}$, we are considering adding a second image intensifier to the $\mathrm{Xybion}$ or switching to an alternative detector to allow $\mathrm{C}^{4+}$ measurements.

\section{CIT DIAGNOSTIC}

By viewing a radially or vertically injected pellet with an array of neutral particle energy analyzers, the spatial profile of the fast alpha energy distribution can be measured on CIT. Spatial resolution is obtained by the intersection of the detector viewing volume and the pellet cloud path. The number of neutral helium atoms per unit energy per unit time produced by fast alphas interacting with the cloud is given by

$$
\frac{d N_{\mathrm{He}^{\circ}}}{d \mathrm{E} d t}=\frac{d n_{\mathrm{He}}++}{d \mathrm{E}} v_{\mathrm{He}}+\mathrm{A}_{\text {cloud }} \mathrm{F}_{0}^{\infty}(\mathrm{E})
$$

where $A_{\text {cloud }}$ is the cross-sectional area of the portion of the cloud dominated by $\mathrm{C}^{4+}$ and observed by the collimated neutral particle energy analyzer. At $3 \mathrm{MeV}$, $\sigma_{20} \sim 3.4 \cdot 10^{-21} \mathrm{~cm}^{2}$ and $\sigma_{01} \sim 1.7 \cdot 10^{-16} \mathrm{~cm}^{-2}$ for a $\mathrm{C}^{4+}$ target so that $\mathrm{F}_{0}^{\infty} \sim 2 \cdot 10^{-5}$. Equation (3) yields a total neutral production of $9 \cdot 10^{15} / \mathrm{sec}$ in a $100 \mathrm{keV}$ energy bin at $3 \mathrm{MeV}$ alpha energy. This assumes an alpha density of $10^{13} \mathrm{~cm}^{-3}$, a classical alpha velocity distribution, and a useful cloud area of only $6 \cdot 10^{-1} \mathrm{~cm}^{2}$. One of the goals of our experiments at TEXT and TFTR is to better determine the useful $\mathrm{C}^{4+}$ cloud area expected for CIT. With a detector solid angle of $3 \cdot 10^{-7}$ (outside the CIT shielding igloo), we can expect to detect $3 \cdot 10^{9}$ neutrals per second in the $3 \mathrm{MeV}$ channel. For a desired radial spatial resolution of $5 \mathrm{~cm}$, the neutral energy analyzer would see neutrals for $\sim 5 \mu \mathrm{sec}$ at a pellet velocity of $10 \mathrm{~km} / \mathrm{sec}$. Hence, we would observe about $1.5 \cdot 10^{4}$ neutrals in this time interval. Lower energy channels would observe a higher 
counting rate due to the energy dependence of $d n_{\mathrm{He}^{+}+} / d \mathrm{E}$ and $\mathrm{F}_{0}^{\infty}(\mathrm{E})$. This neutral signal rate would be well above the expected background rate of $<2 \cdot 10^{7} / \mathrm{sec}$ due to the high neutron and garnma ray flux surrounding CIT.

\section{TFTR DIAGNOSTICS}

D-T operation in TFTR will provide the first opportunity to study alpha particle physics in a tokamak. The pellet velocity needed to penetrate TFTR at $n_{\mathrm{e} 0}=$ $10^{14} \mathrm{~cm}^{-3}$ and $\mathrm{T}_{\mathrm{e}}=10 \mathrm{keV}$ is approximately $5 \mathrm{~km} / \mathrm{sec}$. The alpha density in TFTR will be less than $10^{12} \mathrm{~cm}^{-3}$, but the expected increase in usable cloud size should allow the TFTR signal rate to be within a factor of 10 or less of that previously calculated for CIT. We have begun discussions with PPPL and MIT personnel about collaborating on the impurity pellet injection experiments being performed on TFTR.

\section{SINGLE CHARGE EXCHANGE}

Others ${ }^{13,14}$ have proposed using single charge exchange iriteraction of alphas with the pellet cloud. Photons from the resultant excited helium ions

$$
\begin{gathered}
\mathrm{He}^{++}+\mathrm{C}^{4+} \rightarrow \mathrm{He}^{++}(\text {excited })+\mathrm{C}^{4+} \\
\downarrow \\
\mathrm{He}^{+}+h \nu
\end{gathered}
$$

will be Doppler silifted due to the incident alpha velocity. Unfortunately, the expected signal size is comparable or smaller than the expected background light, e.g., bremsstrahlung continuum from the pellet ablation cloud.

We propose making use of the finite lifetime of the excited helium ions. This lifetime, of the order of a nanosecond, should allow some of the fast alphas to travel out of the plane of the bright cloud before they radiate. Since the ablation cloud is predominately in the plane formed by the pellet velocity and the magnetic field 
direction, a collimated photon detector looking parallel to this plane but a centimeter or so away may avoid the large background light problem.

Another effect ignored in previous papers on the single charge exchange method is the motional Stark effect. Since the alpha velocities and to;oidal magnetic field are both large, the resultant motional Stark effect may complicate or make impossible the use the Doppler shift to determine the velocity distribution of the incident alphas.

\section{CONCLUSIONS}

While we have not been able to directly measure the $\mathrm{C}^{4+}$ spatial distribution, the existing data supports the idea of a large spatial region of the pellet cloud being predominately $\mathrm{C}^{4+}$. This should allow absolute measurement of the fast confined alpha energy distribution in CIT and TFTR using helium neutrals from double charge exchange interactions.

This work was supported by the U.S. Department of Energy under Contract No. DE-AC03-89ER53277. 


\section{REFERENCES}

${ }^{1}$ K. M. Young, Princeton University Plasma Physics Laboratory, AA-870710PPL-06, June 1987.

2 O. N. Jarvis and A. E. Costley, JET Joint Undertaking, JET-P(87)05, December 1986.

3 D. Post, S. Zweben, and L. Grisham, Alpha particle diagnostics, 1986 Varenna Diagnostics School.

${ }^{4}$ S. J. Zweben, Rev. Sci. Instrum. 57, 1723 (1986).

${ }^{5}$ R. K. Fisher, J. S. Leffler, A. M. Howald, and P. B. Parks, Fusion Technology 13, 536 (1988).

${ }^{6}$. S. K. Allison, Rev. Mod. Phys. 30, 1140 (1958).

7 R. Olson, University of Missouri-Rolla, private communication.

${ }^{8}$ P. B. Parks, J. S. Leffler, R. K. Fisher, Nuclear Fusion 28, 477 (1988).

${ }^{9}$ S. Milora, ORNL, private communication.

10 J. E. Osher, et al., IEEE Trans. Plasma Science 17, 392 (1989), and private communications.

11 J. Terry, Massachusetts Institute of Technology, private communication.

12 G. Gerdin, Bull. Am. Phys. Soc. 34, 2032 (1989).

13 M. Sasao, K. N. Sato, W. Wakatani, and Y. Nakamura, Annual Review Nagoya University, Institute of Plasma Physics, April 1983 through March 1984, p. 47.

14 G. Gerdin, Bull. Am. Phys. Soc. 31, 1542 (1986). 

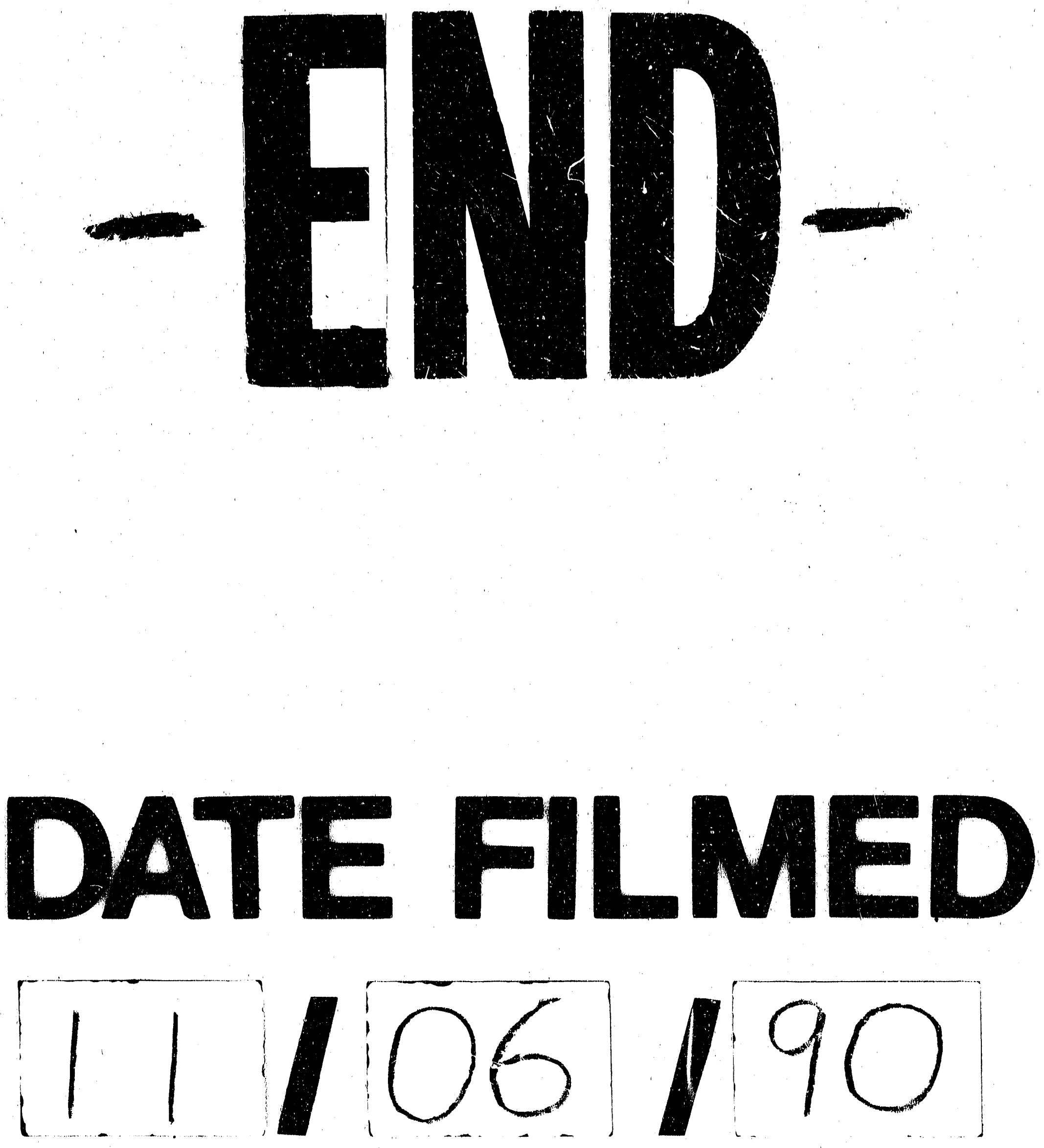
